\title{
Supporting knowledge sharing in heterogeneous Social Network thematic Groups
}

\author{
Antonello Comi*, Lidia Fotia*, Fabrizio Messina ${ }^{\dagger}$, Giuseppe Pappalardo ${ }^{\dagger}$,Domenico Rosaci*, Giuseppe M.L. Sarné * \\ *DIIES Department, University of Reggio Calabria \\ Reggio Calabria, Italy \\ \{antonello.comi,lidia.fotia,domenico.rosaci,sarne\}@unirc.it \\ $\dagger$ DMI Department, University of Catania \\ Catania, Italy \\ \{messina,pappalardo\}@dmi.unict.it
}

\begin{abstract}
Thematic groups are gaining a lot of attention and high centrality in OSNs, as users share opinions and/or mutually collaborate for reaching their targets. Since users can be affiliated with groups belonging to different social networks, they can be supported by personal software agents able to perform activities aimed at supporting relationships and mutual cooperation among heterogeneous OSNs thematic groups. Basically software agents can encode users profiles with detailed information to be related with specific groups. This work discusses a multi-agent framework whose structure focuses on the role of software agents and the use of a common, shared dictionary for each group. Personal software agents are associated to OSN users to share knowledge for a specific theme for a number of groups related to that topic. Group agents are defined to support each group on each OSN by interacting with personal agents to manage group affiliation and enrich the common dictionaries of their own groups. The common dictionary of the group is a key element to provide knowledge sharing and interoperability between personal and group agents. In the proposed approach each user agent is able to personalize its own dictionary and enrich that of its own groups by means of selected categories.
\end{abstract}

Keywords-Online Social Network; Knoledge sharing; Common Dictionary; Intelligent Agents; Thematic Group

\section{INTRODUCTION}

Online Social networks (OSNs) cover, nowadays, almost the totality of the aspects in our life. OSN Thematic groups [1], [2] play an interesting role, because users interested in the same arguments (i.e. literature, movies, computer science, etc.) have the need of sharing opinions and mutually collaborate for reaching their targets (improving knowledge, linking people with the same interests, organizing meetings).

In order to encourage both relationships and cooperation within online social networks groups [3]-[6], many authors investigated just on the nature of such relationships, e.g. those occurring between users and groups [7] and those among the users affiliated with the same group [8]. In particular, several authors highlighted that interactions among users of the same group are complex in nature, and require significant efforts to be carried out by users, particularly for taking into account the different levels of knowledge and the personal representations kept by each group member.

Personal software agents [9] can act in the interest of their owners by avoiding heavy and boring tasks. Employing a personal agent is not generally feasible, because will imply to manage a lot of groups activities also involving topics which are highly different among them. Therefore providing each user with a number of personal agents, where each of them is devoted to observe activities with respect to a specific user's interest or theme (e.g. photography), may represent a more feasible solution. This particular approach allows to design personal agents that support its own user only for a single specific group and therefore agents will be highly specialized. On the other hand there is an emerging problem consisting in providing personal agents with mechanisms aimed at promoting relationships and mutual cooperation within the social network group.

Given the premises above, in this work we present a multiagent framework to support OSNs groups by means of Group Agents which provide some basic services to all its users. Furthermore, a mutual agent understanding is realized by means of a common dictionary storing topics (i.e. names, things, concepts and so on) of interest in each group of the OSN. The dictionary is publicly available by all the agents affiliated with that group, and represents a common knowledge specialized with respect to the matter of discussion for that group. At the same time, personal agents are provided with personal dictionaries, belonging to the analysis of users' behaviors, such that the common, shared dictionary can be enriched by means of the mutual cooperation between personal agents and the agents managing these groups.

The remainder of the paper is as follows. In Section II we introduced the reference scenario, while in Section III the structure of the designed dictionary is discussed. In Sections IV and $\mathrm{V}$ the profiles of the Personal and Group Agents are described. Section VI presents some related literature and the novelties provided by this work. Finally, in Section VII we draw our conclusions and introduce our future works.

\section{The Multi-Agent Social Network INFRASTRUCTURE}

In the proposed model, depicted in Figure 1, we suppose the presence of thematic groups belonging to several different OSNs. Each user $(u)$ that is member of a group $(g)$, is supported in his/her activities by a software agent, called Personal Agent (a). Similarly, each group is managed by a 


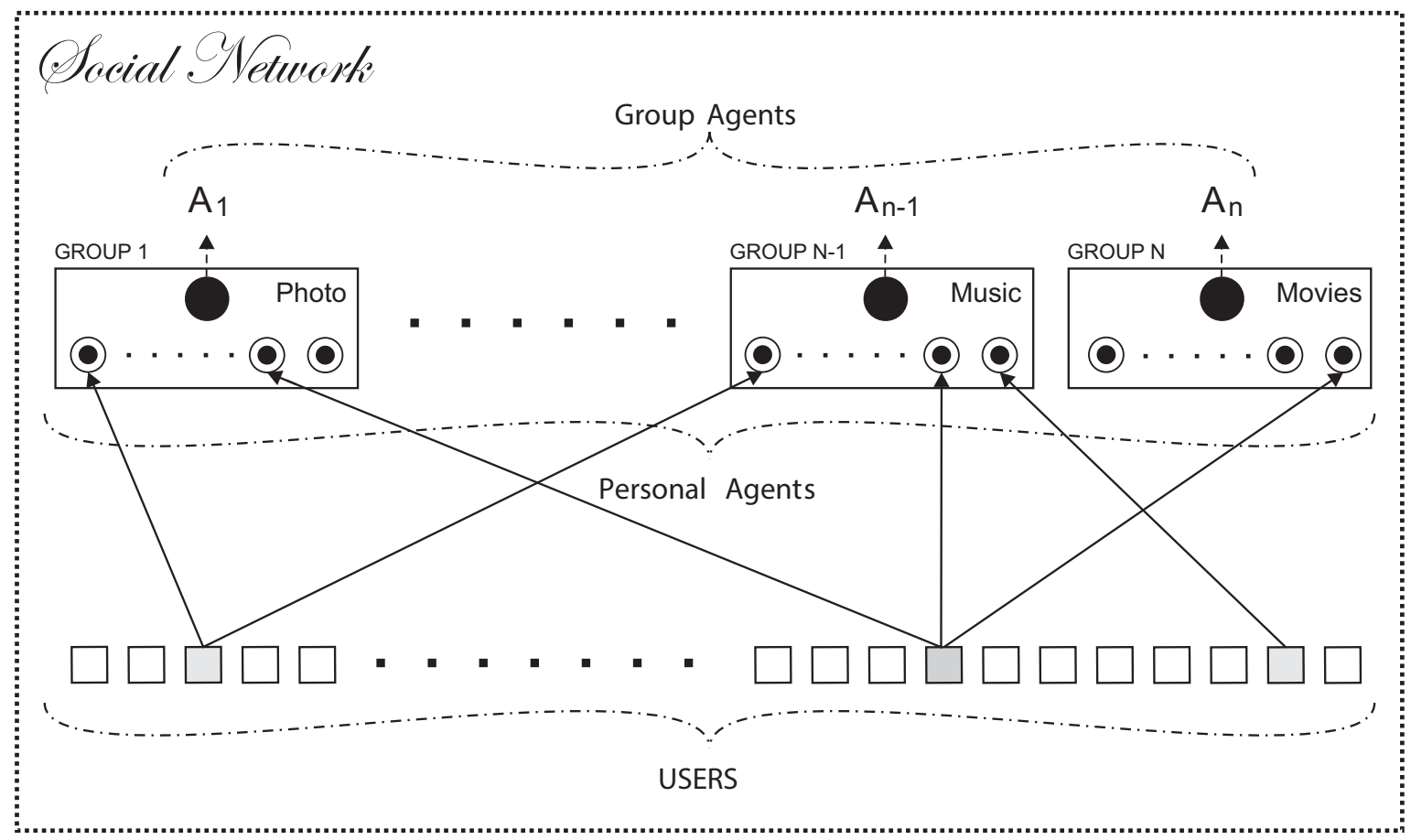

Fig. 1. The proposed Social Network architecture.

a Group Agent ( $A_{i}$ in Figure 1) providing some services to its group users by cooperating with their associated Personal Agents.

As each user can join with multiple thematic groups, he/she will be supported by Personal Agents, one for each theme or topic. Each Personal Agent is devoted to support its user to perform group activities by encoding in its profile all the information needed in order to manage the user interests for the specific topic. As Personal Agents hold, in general, different knowledge, in order to promote mutual agent cooperation it is necessary to provide them with a common knowledge specialized on the focus of that group. For this aim, in the next section we describe a dictionary aimed at describing a common knowledge of all the agents associated with that social network groups that is assumed to be publicly available to all the members (i.e. Personal Agents) associated with that group.

As we discuss later with more details, personal agents are allowed to manage users profiles (i.e. personal dictionaries) with "categories" which currently do not belong to the common dictionary of that group, we call them as "personal" user's categories. Therefore, in order to allow agents interoperability, personal categories should be understandable among all the agents of the same group. To this purpose, we suppose that each personal category is in a general relationship with at least another category belonging to the common dictionary adopted by the agent community of the group.

\section{THE COMMON DiCTIONARY}

In this section we provide a formal description of the Common Thematic Dictionary (CTD), employed to allow Personal Agents to cooperate with their own peers. A CTD is shared among the members of a group, in order to contain the topics (i.e. names, things, concepts and so on) of interest for the group members, as well as the relationships which can occur among such terms. In our approach, the CTD represents the common knowledge of a group; it is publicly available to all the members of the group and extensible (also individually by each Personal Agent) in order to include further knowledge and relationships among old and new entries.

\section{A. Elements of of CTD}

A CTD can be defined as a $i$ ) set $\mathcal{D}_{\mathcal{C}}$ of categories involved in the thematic group and ii) a set of category links $-\mathcal{D}_{\mathcal{R}}$ - which establish relations between the categories belonging to $\mathcal{D}_{\mathcal{C}}$. A category link between two categories $c_{1}, c_{2} \in \mathcal{D}$ is described by a tuple $C L=\left\langle c_{1}, c_{2}, t\right\rangle$, where $t$ is an information denoting what type of link is involved in the relationship existing between the two categories.

We introduce five types of category links, namely basic (B), isa $(\mathrm{I})$, synonymy $(\mathrm{S})$, overlap $(\mathrm{O})$ and cross $(\mathrm{C})$ link types. The first type is associated with all the links, the other three links provide other information exclusively based on the semantic involved in the links and, finally, the last link type cross has been introduced to identify all those terms (i.e. topics) that, although they are not strictly related with the focus of the social network group, they could be debated within the group.

Basic link (B): two categories $c_{1}$ and $c_{2}$ are basic-linked, iff $c_{1}$ is associable with $c_{2}$ with respect to the subject of the CTD. Any other category link is also belonging to this link category. An example is the link connecting the two categories Reflex (camera) and Lens does not belong to any of the other types of considered link categories listed below.

Isa link: two categories $c_{1}$ and $c_{2}$ are isa-linked iff the terms belonging to $c_{1}$ also belong to $c_{2}$. For example, in 
the photography field two categories such as Rangefinder and Camera should be linked by an isa-link, i.e. each rangefinder camera is a camera but not vice versa.

Synonymy Link: two categories $c_{1}$ and $c_{2}$ are synonymylinked iff the terms belonging to $c_{1}$ have the same meaning of those belonging to $c_{2}$. For instance, by remaining in the photography field, two categories such as Picture and Image should be considered as synonymy-linked because $c_{1}$ and $c_{2}$ correspond as meaning. It avoids the problem of renaming an existing category in order to take into account the ambiguity of the natural language which might exploit different terms for identifying the same object, concept, etc.

Overlap Link: two categories $c_{1}$ and $c_{2}$ are overlap-linked, iff some terms of $c_{1}$ also belong to $c_{2}$, and vice versa. For instance, if we consider the two categories Daguerreotype and Antique, they might be linked by an overlap-link. Indeed, the first term identifies an image realized with the old daguerreotype process and it is possible (i.e. probable) that it is also an antique image but it is also true that an antique image might be a daguerreotype. Note that when two categories are synonymy-linked, they are also overlap-linked.

Cross link: two categories $c_{1}$ and $c_{2}$ are cross-linked iff it is supposed that members of a group that are interested in $c_{1}$ are usually interested also in $c_{2}$. For instance, two categories such as Picture and Video might be considered as cross-linked since many modern cameras can also realize videos and, therefore, it can be supposed that many users interested in the photography might be also interested in videos or, more in general, in other categories sufficiently closed to the focus of the group as the example above. In particular, in this case if the agent owner is affiliated with only the group dealing with the photography his/her agent will provide to manage also information about video, conversely if this user is also affiliated with the group dealing with videos then both his/her agents will cooperate about the matter of the cross-link topics in the interest of their common owner.

\section{B. Representation of the CTD}

A CTD $\mathcal{D}$ can be represented by a direct graph $G_{\mathcal{D}}=$ $\left\langle\mathcal{D}_{C}, \mathcal{D}_{R}\right\rangle$, where $\mathcal{D}_{C}$ represents the set of nodes, i.e. the categories. Similarly, $\mathcal{D}_{R}$ is the set of arcs of the graph, which is represented by the links $\left\langle c_{1}, c_{2}, t\right\rangle \in \mathcal{D}_{R}$, with $t \in\{B, I, S, O, C\}$, as explained in Section III-A. Note that arcs associated with an isa link type are depicted with orientation, because $\left\langle c_{1}, c_{2}, I\right\rangle$ does not imply $\neq\left\langle c_{2}, c_{1}, I\right\rangle$, while arcs with the other link types (e.g. synonymy, overlap, cross and basic), for which $\left\langle c_{1}, c_{2}, t\right\rangle \equiv\left\langle c_{1}, c_{2}, t\right\rangle$, for convenience are depicted without orientation ${ }^{1}$. Figure 2 shows an example of CTD concerning Photography. In particular, links that belong to more than one category have multiple labels, e.g. $\langle$ Picture, Image, $(B, S, O)\rangle$.

Moreover, we say that two categories $c_{1}$ and $c_{2}$ are in a t-relationships when in $\mathcal{D}$ there exists a path $\left\langle c_{1}, c_{k}, t\right\rangle \ldots\left\langle c_{l}, c_{2}, t\right\rangle$. Differently, if the links of this path joining the nodes $c_{1}$ and $c_{2}$ belong to different category links we say that they are generally related.

\footnotetext{
${ }^{1}$ In the following of this paper, we will refer to the CTD as $G_{\mathcal{D}}$ or $\mathcal{D}$ in an interchangeable manner
}

\section{The Personal Agent}

In the proposed model each OSN user $u_{i}$ is assisted by a number of Personal Agents $\left(a_{i 1}, a_{i 2}, \ldots, a_{i g_{i}}\right)$, where $g_{i}$ is the number of groups to which the user $u$ have joined. Formally, for each group $k$ to which the user $u_{i}$ is affiliated to, it will exist an agent $a_{i j}$ which manages the affiliation of the user with that group and a number of other groups with the same theme. Therefore, each Personal Agent focuses on the activities related of its own user and a specific theme.

User Profile. Each agent is able to maintain a detailed profile $P$ by analyzing the activities of its own user into the OSN. In order to represent the profile $P$ for a user $u_{k}$, the same notation of CTD is adopted, i.e. a graph $G_{k}=\left\langle P_{C}^{k}, P_{R}^{k}\right\rangle$ where $P_{C}^{i}$ is the set of categories and $P_{R}^{k}$ is the set or category links. Each category $c \in \mathcal{P}_{\mathcal{C}}$, which is of interest for the user, is represented by a tuple $\Gamma_{c}=\left\langle i_{c}\right.$, mode $\left.e_{c}\right\rangle$, where $i \in[0,1] \subset \mathbb{R}$ represents the level of interest of the user for the category $c$ $\left(0 / 1\right.$ denotes the minimum/maximum interest for $c$, and mode $_{c}$ specifies the level of visibility that $u$ desires to give to his/her own interest for $c$; it can assume values public or private. Figure 3 reports an example of agent profile which is derived, for convenience, from the example CTD proposed in Figure 2. Please note that three categories (filled in gray) are not depicted into figure 2 .

Analyzing category interest. The Personal Agent will manage the "personal" categories of interest for its own user by monitoring the user's behavior when he/she interacts within the group. Let be $\gamma_{k} \in \mathbb{N}^{+}$a threshold, and $S_{k}=\left\{c_{1}, c_{2}, \ldots, c_{\gamma_{k}}\right\}$ a set of categories $c \in P_{C}^{k}$ having the higher index $i_{c}$, i.e. for which the user has shown the higher level of interest. The Personal Agent periodically updates the set $S_{k}$, and computes the coefficient $m_{k, D_{j}}$ for all the dictionaries $D_{j}$ of the groups having the topic (theme) of interest of the user $u_{k}$ :

$$
m_{k, D_{j}}=\frac{1}{\left\|S_{k} \cap D_{j}\right\|} \sum_{c \in\left\{S_{k} \cap D_{j}\right\}} \frac{i_{c}}{i_{\max }}
$$

where $i_{\max }$ is the maximum level of interest and $\left\{S_{k} \cap D_{j}\right\}$ is the set of categories which are in common between the personal dictionary of the user $u_{k}$ and the CTD $D_{j}$.

The ratio behind computation of index $m_{i, D_{k}}$ is simple. As the interest of the user for some categories decreases, the number $i_{c}$ for the corresponding categories will fall down, therefore, $m_{k, D_{j}}$ will decrease. Conversely, for any group $j$ concerning the same topic managed by the agent $a_{k}$, values of $m_{k, D_{j}}$ will increase. Index $m_{k, D_{j}}$ is used by the Personal Agent to manage group affiliation, as explained below.

Group affiliation. The Personal Agent is delegated to suggest a group which may be of interest of its own user and/or sending an affiliation request to any Group Agent. Conversely, it will suggest to leave a group and/or send a leave message to the Group Agent for which the interest of the user is low.

The Personal Agent is delegated to retrieve all the CTD of the groups having the same topics of interest which is of its own responsibility. Index $m_{k, D_{j}}$ is computed for all those 


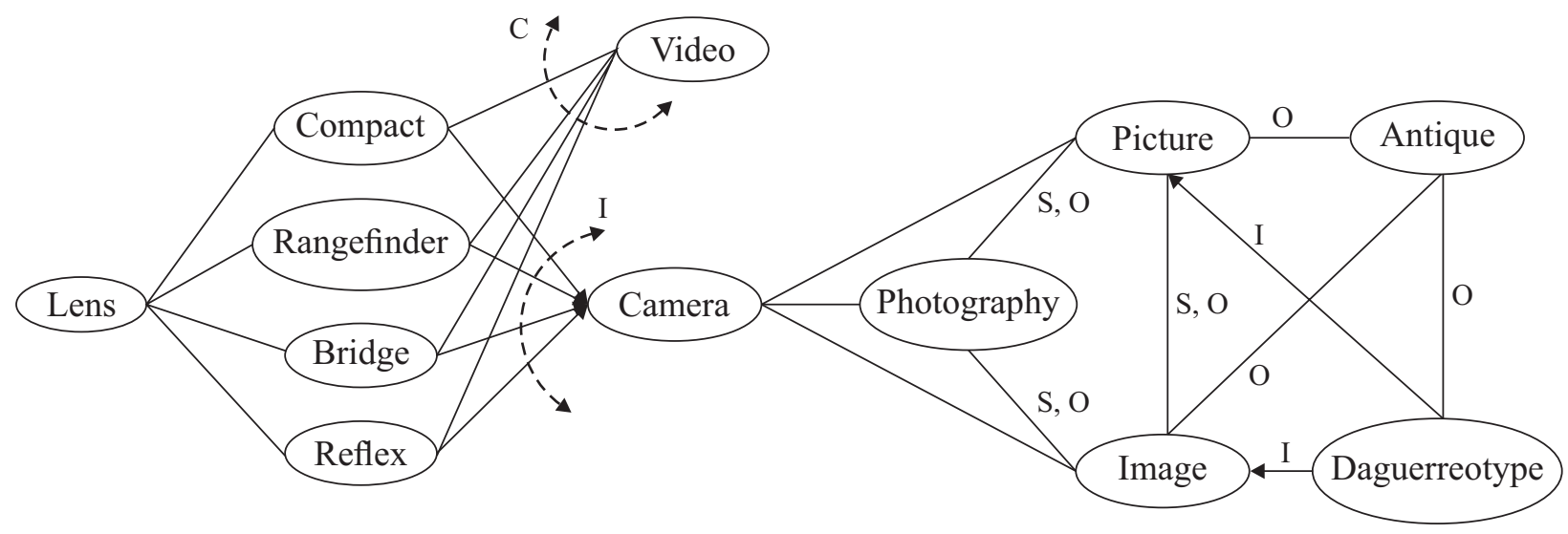

Fig. 2. A part of a Common Thematic Dictionary concerning Photography (the basic link type specification is omitted since all the links are always basic links).

groups, and is used to select "group candidate", which may be of interest for the user.

Therefore, a threshold $\eta \in[0,1] \in \mathbb{R}$ is fixed by the Personal Agent such that, when $m_{m_{k}, D_{j}^{*}}>\eta$ for a group $j^{*}$ to which the user does not belong to, group $j^{*}$ becomes a candidate for the user to join with. Similarly, once it is verified that $m_{k, D_{\widehat{j}}}<\eta$ for a group $\widehat{j}$ (i.e. a CTD $D_{\widehat{j}}$ ) of which the user is part, the Personal Agent $a_{k}$ will suggest to the user to leave the group $\widehat{j}$ or will send a leave request to the Group Agent of group $\hat{j}$.

Matching category links. A further comparison is performed by the Personal Agent on existing connections between categories $c_{1} \in\left\{S_{k} \cap D_{j}\right\}$ and $c_{2} \in\left\{S_{k}-D_{j}\right\}$, by considering the category links basic, synonymy and overlap. In particular, we denote by $N_{s}\left(c_{1}, c_{2}\right)$ the number of connections of type $S$ (Synonymy), $N_{o}\left(c_{1}, c_{2}\right)$ the number of connections of type $O$ (Overlap) and $N_{b}\left(c_{1}, c_{2}\right)$ the number of connections of type $B$ (Basic), between $c_{1}$ and $c_{2}$ :

$L_{k, D_{j}}\left(c_{1}, c_{2}\right)=\frac{w_{s} N_{s}\left(c_{1}, c_{2}\right)+w_{o} N_{o}\left(n_{1}, n_{2}\right)+w_{b} N_{b}\left(c_{1}, c_{2}\right)}{N_{c_{2}}}$

where $c_{1} \in\left\{S_{k} \cap D_{j}\right\}, c_{2} \in\left\{S_{k}-D_{j}\right\}, w_{s}, w_{o}$, and $w_{b} \in$ $[0,1] \subset \mathbb{R}, \sum w=1$ and $w_{s} \geq w_{o} \geq w_{b}$. The last choice is due to the fact that a synonymy link is semantically stronger than the overlap one, which, in turn, is stronger than the basic one. Finally, $N_{c_{2}}$ is the total number of links of the category $c_{2} \in\left\{S_{k}-D_{j}\right\}$. Since $N_{s}+N_{o}+N_{b} \leq N_{c_{2}}$, it will be $L_{i, D_{k}}<1$.

The aim of the computation of $L_{k, D_{j}}$ is to select categories belonging to users dictionaries, in order to enrich the CTD of the group with users categories, as explained below.

Dictionary enrichment. Let be $j^{*}$ a group to which user $u_{k}$ is affiliated. First of all, Personal Agent $a_{k}$ will compute index $L_{k, D_{j}}$ for all $c_{1} \in\left\{S_{k} \cap D_{j}\right\}$ and $c_{2} \in\left\{S_{k}-D_{j}\right\}$. Afterwards, for all $c_{2} \in\left\{S_{k}-D_{j}\right\}$, the following computation is performed

$$
\widehat{L}_{k, D_{j}}\left(c_{2}\right)=\frac{1}{\left|S_{k} \cap D_{j}\right|} \sum_{c \in S_{k} \cap D_{j}} L_{k, D_{j}}(c, c 2)
$$

A threshold $\theta \in[0,1] \subset \mathbb{R}$ is fixed by Personal Agent $a_{k}$ such that, when $\widehat{L}\left(c_{2}\right) \geq \theta$, category $c_{2} \in\left\{S_{k}-D_{j}\right\}$ is sent to the group agent to enrich the CTD of the group. As we explain in Section $\mathrm{V}$, once the group agent has received such requests, it will be consider to enrich the CTD of its own group by selecting other parameters, as frequency of the involved terms.

\section{The Group Agent}

Group Agents interface with the community of Personal Agents (i.e users) for group affiliation and CTD management. Fir this aim, Group Agent manages some data structures to encode the profile of the group.

In particular, as described in Section III, the Common Thematic Dictionary (CTD) of the group is able to store all the topics of interest for the group members, as well as relationships possibly occurring among such terms (see Section III). The White Pages service stores the identifiers of all the affiliated agents. Finally, the public interests of the group members are stored in a third data structure simply denoted as Yellow Pages. By means of yellow pages, agents can identify other agents (i.e. users) in the group that have interest in its same topics. This data structure is organized as set of lists, each of them associated with a user (i.e. agent) and affiliated with the group.

\section{A. Group Agent behavior}

Group affiliation. When a user joins the group managed by the Group Agent, a new identifier is provided to the newcomer, and White Pages are updated. Conversely, once a user wants to leave the group, the Group Agent provides to delete all the associated information from its own data structures.

Dictionary enrichment. The CTD of the group is periodically updated basing on two main information. The first 


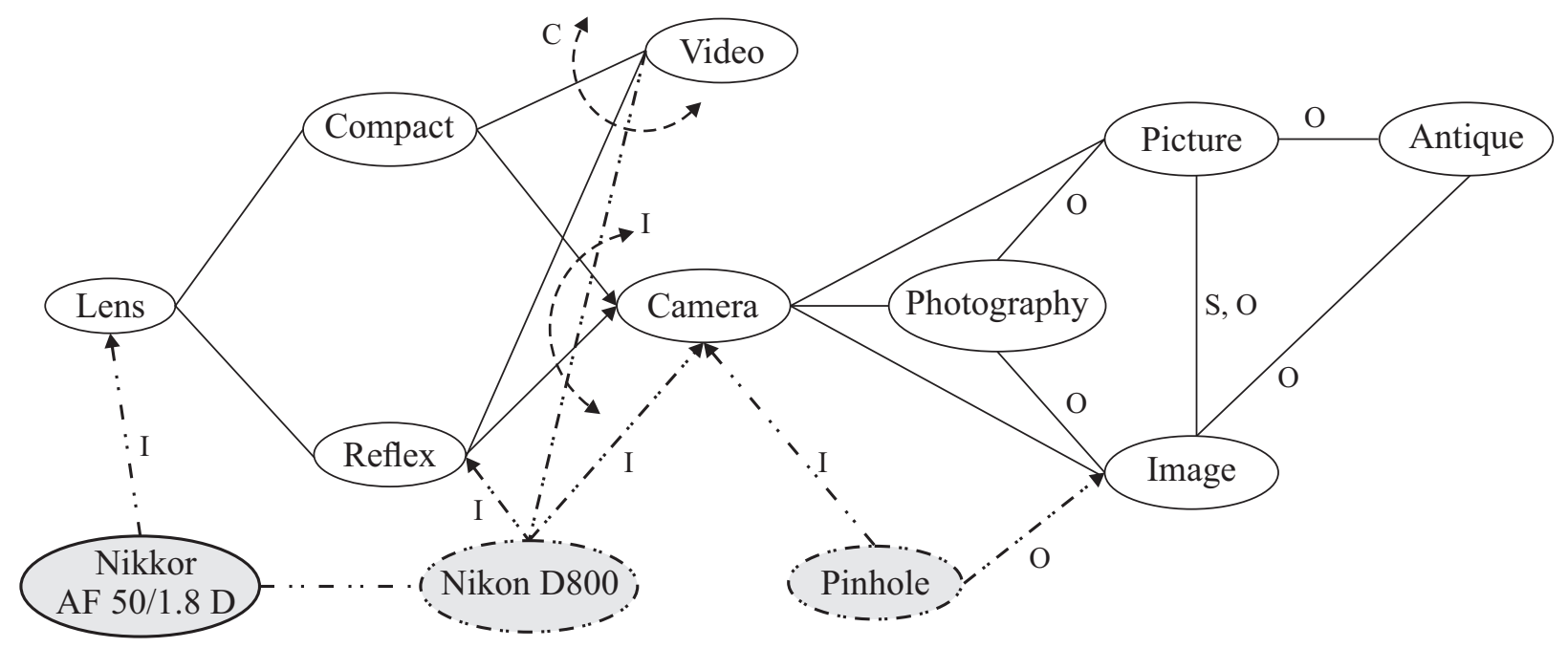

Fig. 3. An example of Personal Agent profile based on the CTD of Figure 2 (the basic link type specification is omitted; in grey the added personal knowledge).

is the the knowledge of the affiliated Personal Agents (see Section IV). To this aim the Group Agent will collect all the categories $c \notin D_{j}$ which are sent by Personal Agents $a_{i}$ due to the fact that index $\widehat{L}_{k, D_{j}}>\theta_{i}$ (see Section IV). We remember that agent $a_{k}$ is interested to enrich the dictionary of the group with category $c \in D_{j}$ because $i$ ) category $c \in S_{j}$, i.e. is in the set for which user has an high interest $i_{c}$ and $\left.i i\right)$ $c$ is "quite connected" with other categories belonging to the set $\left\{S_{i} \cap D_{k}\right\}$. The information above $\left(L_{k, D_{j}}\right)$ will provide to the Group Agent a first set of candidate categories. From this set the Group Agent will take into account the set of categories having the highest frequency $f^{2}$, i.e. which are sent by the higher number of Personal Agents, such that the CTD is enriched with further knowledge which is shared among the groups members.

\section{RELATED WORK}

A great number of researchers investigated on the topic embraced in this work, therefore, in this section we cite only those approaches which we consider significant with respect to that discussed in this paper.

The agent ability of collaborating with other agents generally implies their mutual understanding ability, which can be realized by exploiting some form of common knowledge shared by all the cooperating agents [10]. For instance, authors of [1] present a multi agent system on which agents share a common hierarchical ontology able to represent their interests. Differently from our proposal, their approach does not allow agents to represent their private knowledge. This is a strong limitation for those agents which have to provide their owners with a specific support. To avoid such limits, in [11] it is proposed the use of a dictionary expansible with individual knowledge. As in our approach, graphs are used to represent the common knowledge and the agent profiles although profiles information are more structured and specialized that in our proposal.

\footnotetext{
${ }^{2}$ The frequency is computed among all the used terms which fall into those categories
}

Other approaches do not need a common and shared ontology, for instance the proposal presented in [12] adopts a meta-ontology to translate the presuppositions extracted by a message of a sender agent in a form understandable from the receiver agent. For performing this translation a vocabulary whose terms are understandable to both the agents is exploited. However, once the receiver agent has identified inconsistencies, type conflicts or ontological gaps, it provides to modify its personal ontology in a form compatible with the required understanding of the incoming message.

Similarly, in [13] a domain-specific ontology, called global ontology, is used by agents along with a matchmaking system used to avoid the adoption of shared ontologies. In particular, each agent which is joined with the platform provides to map its personal ontology into the global one. This process is performed by an information-extraction engine able to identify relevant information as concept names (e.g. class names, parameter names, etc.) adopted into the personal agent ontology. As a result, there is not a shared common ontology which all the agents have to adopt but another one which is used for translation purpose only.

To deal with potential heterogeneities existing in digital libraries, the authors of [14] designed a P2P agent framework where each library is associated with a software agent in order to realize a common dictionary (i.e. ontology) to support semantical agent communication. A technique based on the use of shared keys, semantically negotiated by agents, is proposed in [15] to solve the synonym problem for avoiding to adopt different terms in describing the same objects.

Another mechanism for assuring agents interoperability is that of exploiting the semantic negotiation. A framework based on the semantic negotiation is provided in [16]. More in particular, agents searching the meanings of unknown terms engage collaborative activities with other agents they consider more expert. In such a way, it is obtained a form of centralized knowledge in a form of dictionary or ontologies. While this approach results more versatile with respect to dictionaries and ontologies which necessarily require to codify the knowledge, from the other hand it is more expansive under a computational 
and a communication point of view.

The presented approaches support mutual collaboration among software agents by promoting their mutual understanding and the most part of them are based on the use of some form of shared knowledge, i.e. dictionaries, common ontologies or a global ontologies, while in [16] other agents are considered as experts in the involved domain and having similar ontologies.

The approach discussed in this paper is based on a versatile form of dictionary and, in our opinion, should result more simple and effective that the other proposals. Moreover, our dictionary have been natively designed to be enriched by agents in order to adapt the common and the personal knowledges to the evolution of groups without to require complex procedures. However, such supposed performances of our framework should be verified by testing its prototype that we are implementing.

\section{CONCLUSIONS AND FUTURE WORK}

In this paper we dealt with the problem of promoting users interactions and mutual cooperation within social network thematic groups by designing a multiagent framework. In particular our approach allows us to take into account the presence of heterogeneous knowledge among group members (i.e. agents).

To this purpose, in our framework each thematic group is supported by a Group Agent and, in turn, each affiliated group user is supported by a Personal Agent. More in detail, each Group Agent provides some basic services to each of its affiliated Personal Agents. Each of these Personal Agents is specialized only to handle just with a specific them, and manages a personal dictionary of user's knowledge and interest. As a consequence, users are supported by a variable number of Personal Agents, one for each theme of interest for the user.

In the proposed approach, such Personal Agents observe user behaviors in order to update the personal dictionaries of their own users. As group dictionaries (CTD) have been designed to be extensible by each Personal Agent with other topics of interest, Personal Agents are able to compute some indexes aimed at enriching common dictionaries of thematic groups to which they are affiliated.

For our ongoing researches we are designing a detailed simulation [17], [18] of our multiagent framework realizing all the functionalities described in this paper, in order to verify how this framework can give to the group users a real advantage in terms of efficiency and effectiveness. The details of the simulation, as well as the obtained experimental results, will be dealt with in a future work.

\section{ACKNOWLEDGMENT}

This work has been supported by project PRISMA PON04a $2 \mathrm{~A} / \mathrm{F}$ funded by the Italian Ministry of University and NeCS Laboratory of the Department DICEAM, University Mediterranea of Reggio Calabria.

\section{REFERENCES}

[1] F. Buccafurri, D. Rosaci, G. M. L. Sarné, and L. Palopoli, "Modeling cooperation in multi-agent communities," Cognitive Systems Research, vol. 5, no. 3, pp. 171-190, 2004.

[2] S. Gauch, M. Speretta, A. Chandramouli, and A. Micarelli, "User profiles for personalized information access," in The Adaptive Web, ser. LNCS, vol. 4321. Springer, 2007, pp. 54-89.

[3] F. Messina, G. Pappalardo, D. Rosaci, C. Santoro, and G. M. L. Sarné, "HySoN: A Distributed Agent-Based Protocol for Group Formation in Online Social Networks," in Multiagent System Technologies. Springer, 2013, pp. 320-333.

[4] P. De Meo, F. Messina, D. Rosaci, and G. M. Sarne, "Improving the compactness in social network thematic groups by exploiting a multi-dimensional user-to-group matching algorithm," in Intelligent Networking and Collaborative Systems (INCoS), 2014 International Conference on. IEEE, 2014, pp. 57-64.

[5] P. De Meo, F. Messina, D. Rosaci, and G. M. L. Sarné, "2dsocialnetworks: Away to virally distribute popular information avoiding spam," in Intelligent Distributed Computing VIII. Springer International Publishing, 2015, pp. 369-375.

[6] P. De Meo, F. Messina, D. Rosaci, and G. M. Sarné, "Recommending users in social networks by integrating local and global reputation," in Internet and Distributed Computing Systems. Springer International Publishing, 2014, pp. 437-446.

[7] Z. Wang, D. Zhang, X. Zhou, D. Yang, and Z. Yu, "Discovering and profiling overlapping communities in location-based social networks," Trans. Sys. Man Cyber: Systems, vol. 44, no. 4, pp. 499-509, 2014.

[8] K. Osipov and G. Sukthankar, "Forming effective teams from agents with diverse skill sets," in Social Informatics (SocialInformatics), 2012 International Conference on. IEEE, 2012, pp. 44-48.

[9] M. Wooldridge and N. R. Jennings, "Intelligent agents: Theory and practice," The knowledge engineering review, vol. 10, no. 02, pp. 115$152,1995$.

[10] J. Waters, B. J. Powers, and M. G. Ceruti, "Global interoperability using semantics, standards, science and technology (gis 3 t)," Computer Standards \& Interfaces, vol. 31, no. 6, pp. 1158-1166, 2009.

[11] L. Palopoli, D. Rosaci, and G. M. L. Sarné, "Introducing specialization in e-commerce recommender systems," Concurrent Engineering: Research and Applications, vol. 21, no. 3, pp. 187-196, 2013.

[12] R. Beun, R. van Eijk, and H. Prust, "Ontological Feedback in Multiagent Systems,' in AAMAS '04: Proceedings of the 3rd International Joint Conference on Autonomous Agents and Multiagent Systems. Washington, DC, U: IEEE Computer Society, 2004, pp. 110-117.

[13] D. Embley, "Toward Semantic Understanding: An Approach Based on Information Extraction Ontologies," in CRPIT 04: Proceedings of the 15th Australasian Database Conference, Volume 27. Australian Computer Society, 2004, pp. 3-12.

[14] H. Ding and I. Sølvberg, "Towards the Schema Heterogeneity in Distributed Digital Libraries," in ICEIS (5), 2004, pp. 307-312.

[15] R. Guha, "Semantic Negotiation: Co-identifying Objects Across Data Sources," in AAAI '04 Spring Symposium Series: Proceedings of the Semantic Web Services, March 2004.

[16] S. Garruzzo and D. Rosaci, "Ontology enrichment in multi agent systems through semantic negotiation," in On the Move to Meaningful Internet Systems 2007: CoopIS, DOA, ODBASE, GADA, and IS. Springer, 2007, pp. 391-398.

[17] F. Messina, G. Pappalardo, and C. Santoro, "Complexsim: an smpaware complex network simulation framework," in Proceedings of Complex, Intelligent and Software Intensive Systems (CISIS), 2012 Sixth International Conference on. IEEE, 2012, pp. 861-866.

[18] — "Complexsim: a Flexible Simulation Platform for Complex Systems," International Journal of Simulation and Process Modelling, vol. 8, no. 4, pp. 202-211, 2013. 